\title{
STATISTICAL FORMULATION AND EXPERIMENTAL DETERMINATION OF GROWTH RATE OF MICROMETRE CRACKS UNDER IMPACT LOADING
}

\author{
WFNSHENG HAN, MFNGFEN XIA*, I.FTIAN SHFN and \\ YILONG BAI \\ LNM, Institute of Mechanics, Chinese Academy of Sciences, Beijing 100080, China
}

(Received 23 December 1994 ; in revised form 4 March 1996)

\begin{abstract}
A new statistical formulation and a relevant experimental approach to determine the growth rate of microcracks were proposed. The method consists of experimental measurements and a statistical analysis on the basis of the conservation law of number density of microcracks in phase space. As a practical example of the method, the growth rate of microcracks appearing in an aluminium alloy subjected to planar impact loading was determined to be $c a .10 \mu \mathrm{m} / \mu$ s under a tensile stress of $1470 \mathrm{MPa}$ and load duration between $0.26 \mu \mathrm{s}$ and $0.80 \mu \mathrm{s}$. $C 1997$ Elsevier Science Ltd.
\end{abstract}

\section{INTRODUCTION}

The growth of cracks in materials under stress has been widely studied in engineering. Some new results, such as the existence of a critical crack propagation velocity much lower than the Rayleigh wave speed, have been obtained in recent years (Fineberg et al., 1992; Shioya, 1993). Gurson (1975) provided a useful model of damaged media, by averaging the effects of many microvoids and considering the presence and growth of a single void in a cell. Some significant advances in material failure modelling have been made with the aid of the Gurson model, e.g. Needleman and Tvergaard (1992). The growth of a single crack under impact loading has been explored by Prakash et al. (1992) and Freund (1992). Until now there have been very few fundamental investigations of the growth rate of micrometre cracks in materials subjected to impact loading. Curran et al. (1987) have studied the failure of materials involving the nucleation, growth and coalescence of microcracks, and referred to their approach as "microstatistical fracture mechanics". In order to study this transient phenomenon, Curran et al. (1987) suggested a "frozen in" experimental technique and an iterative procedure to deduce the crack growth rate. Their procedure of measuring crack growth rates involved indirect inference and is summarized as follows. Firstly, obtain an estimate for the crack growth rate. The next step is to use constitutive relations of the resultant damage and perform computational simulations. Finally, by comparing the computational and experimental results the crack growth rate is modified until a satisfactory agreement is achieved. In the present manuscript, using a model based on the conservation of the number density of microcracks in phase space, an experimental approach to determine the growth rate of microcracks is presented. The result shows that the growth rate of microcracks in an aluminium alloy is a function of the incremental crack extension, and is approximately $10 \mu \mathrm{m} / \mu \mathrm{s}$ under a tensile stress of $1470 \mathrm{MPs}$, within $0.8 \mu \mathrm{s}$.

\section{BASIC EQUATIONS AND FORMULATION}

The essential idea of experimental determination of the growth rate of micrometre cracks under impact loading is to derive the crack growth rate based upon the evolving distributions of microcracks in association with the fundamental equations governing the

* Permanent address: Department of Physics, Peking University, Beijing 100871, China. 
evolution of number density of microcracks. This section provides the necessary background including the basic equations and relevant formulations.

The conservation law of microcracks in phase-space leads to the following general evolutionary equation for the number density of microcracks Bai et al., 1991.

$$
\frac{\partial n}{\partial t}+\sum_{i=1}^{1} \frac{\partial\left(n P_{i}\right)}{\partial p_{i}}=n_{\mathrm{N}}-n_{\mathrm{A}}
$$

where $n\left(p_{1}, p_{2}, \ldots, p_{I}\right)$ is the number density of microcracks. More concretely $n\left(p_{1}, p_{2}, \ldots, p_{I}\right) \mathrm{d} p_{1} \mathrm{~d} p_{2} \ldots \mathrm{d} p_{I}$ is the number of microcracks in the volume $\mathrm{d} p_{1} \mathrm{~d} p_{2} \ldots \mathrm{d} p_{I}$. The coordinates $p_{1}, p_{2}, \ldots, p_{I}$ are the necessary variables constituting an $I$-dimensional phase space and describing the states of microcracks, e.g. sizes, orientations and spatial coordinates. So, different states of microcracks can be mathematically described by a point in the phase space formed by $p_{i}$ coordinates. This space is called the phase space of microcracks; $t$ is the generalized time. $P_{i}=\dot{p}_{i} \neq 0$ is the rate of a sensitive variable of microcracks $p_{i}$, e.g. microcrack length. $n_{\mathrm{N}}$ and $n_{\mathrm{A}}$ are nucleation and annihilation rates of number density of microcracks, respectively.

The effect of interaction of microcracks on the evolution of damage is twofold. Firstly, every microcrack may alter the stress field within which other microcracks nucleate and grow. We shall explain this matter later. The second is of coalescence of microcracks. Obviously, two microcracks can coalesce when a threshold condition is satisfied. A cascade of coalescence of microcracks from small to large scales may lead to complete failure. This evolution induced catastrophe (EIC) presents some unforseen complexity, such as samplespecific behaviour and breakdown of scaling law. Discussions on these issues are beyond the scope of this paper. Readers interested in this matter can refer to Bai et al. (1994a; 1994b), Xia et al. (1994). In the present study, we neglect the coalescence of microcracks and assume their annihilation, $n_{\mathrm{A}}=0$. Our experimental measurement in Section 4 confirms this assumption.

Figure 1 shows a micrograph of microcracks appearing in an aluminium alloy (Al$\mathrm{Cu}$ ) subjected to planar impact. In fact, in planar impact tests, most of the microcracks are approximately penny-shaped and perpendicular to the loading direction (Curran et al., 1987), i.e. these microcracks, seen from Fig. 1, were cut from penny-shaped microcracks inside the material and most of them were parallel with each other. Thus, their size plays the most significant role in damage evolution. The variation of orientations of microcracks can be neglected. Therefore, we consider the size of microcracks as the variable describing the microcracks. Hence, the number of microcracks $n\left(p_{1}, p_{2}, \ldots, p_{l}\right) \mathrm{d} p_{1} \mathrm{~d} p_{2} \ldots \mathrm{d} p_{I}$ is simplified by $n(c) \mathrm{d} c$, where $c$ is the length of microcracks at this time. That is, $n(c) \mathrm{d} c$ is the number of microcracks with a length $c \rightarrow c+\mathrm{d} c$. Therefore, the equation for evolution of microcracks, in one-dimensional phase space, can be expressed as (Xing, 1986; Bai et al., 1988; Ke et al., 1990),

$$
\frac{\partial n}{\partial t}+\frac{\partial(n A)}{\partial c}=n_{\mathrm{N}}
$$

where $A=\dot{c}$ is the average crack growth rate. In the model, $A$ could be either positive or negative, corresponding to growth or healing of microcracks, respectively. In the planar impact experiment, only growth of microcracks was observed, since unloading "freezes" the microcracks in the specimen. As mentioned before, eqn (2) has neglected the coalescence of microcracks. In fact, at the initial or even intermediate stage of damage evolution, the probability of coalescence of microcracks is small. Furthermore, as many researchers have pointed out, the nucleation and growth rates are a function of the sizes of microdamage and macroscopic stress based upon their experimental experiences (Curran et al., 1987). The variation of stress field owing to nucleation and growth of microcracks can be included in the equation in terms of mean stress field. Therefore, we define microcracks in such a 


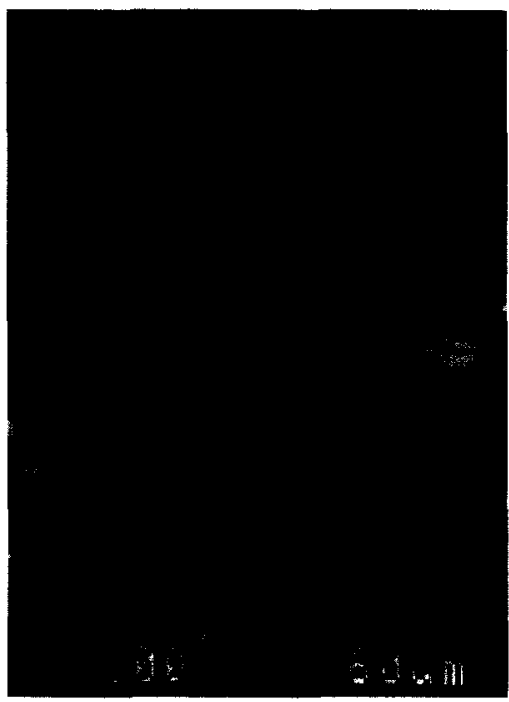

Fig. 1. Microcracks formed in specimens under impact loading. 


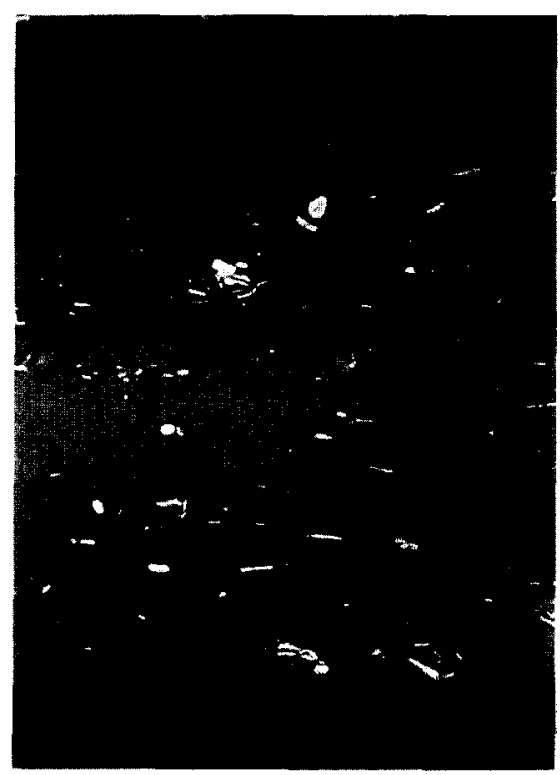

(a)

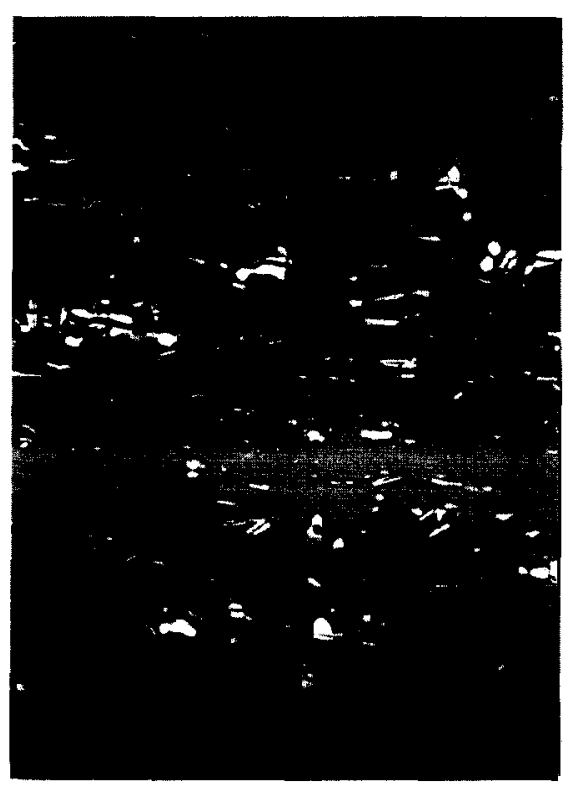

(c)

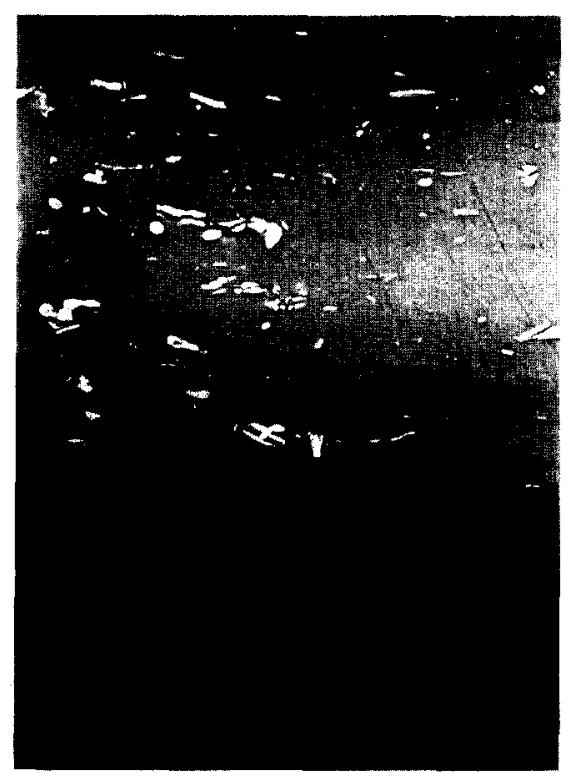

(b)

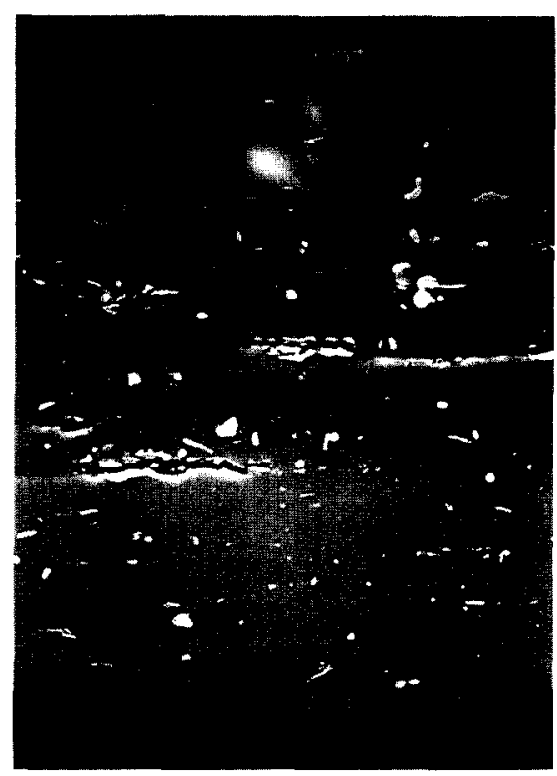

(d)

Fig. 4. Sequential pictures of microcracks appearing in an aluminium alloy (Al-Cu) subjected to planar impact $(\sigma \sim 1.5 \mathrm{GPa}$, time, $t$ is (a) $<0.1 \mu \mathrm{s}$; (b) $0.26 \mu \mathrm{s}$; (c) $0.5 \mu \mathrm{s}$; (d) $0.8 \mu \mathrm{s}$ ). 
system as ideal microcracks. Speaking more clearly, the system of ideal microcracks implies that:

(1) Coalescence of microcracks is neglected.

(2) Nucleation and growth of microcracks are independent of each other.

(3) Nucleation and growth of a microcrack is uniquely governed by a macroscopic stress field.

(4) Interaction between microcracks can be considered in terms of the average variation of the stress field.

Obviously, the model of ideal microcracks corresponds to initial and possibly, intermediate regimes of damage. In order to visualize the model, let us examine some experimental data. The tested aluminium alloy has $\mathrm{Al}-\mathrm{Cu}$ second phase particles of about $3 \times 10^{3} / \mathrm{mm}^{2}$ and approximately $4 \%$ area fraction. When approaching macroscopic fracture, $t=1 \mu \mathrm{s}$ under a tensile stress of about $1.5 \mathrm{GPa}$ (Shen et al., 1992), cracked second phase particles are about $1 \times 10^{3} / \mathrm{mm}^{2}$. So, even just before critical failure, microcracks remain quite sparse. Numerical simulations demonstrate similar sparse distribution of microcracks at EIC. In Section 4, we will provide another experimental verification of the ideal microcrack model. In the model of ideal microcracks the variation of the average stress field due to interaction of microcracks is included in the equation. This leads to two dynamic laws,

$$
\begin{gathered}
n_{\mathrm{N}}=n_{\mathrm{N}}\left(t, c, \sigma_{0}\right) \\
A=A\left(t, c, \sigma_{0}\right)
\end{gathered}
$$

where $\sigma_{0}$ represents the remove average stress and can be a function of damage, $\sigma_{0}=\sigma_{0}$ $(n)$, this equation is similar to the concept of the valid stress of continuum mechanics, the number density of microcracks is similar to the damage variable. $\Lambda$ s shown in Section 5 , for our studied case, the damage fraction is still not very large, and the stress approximately holds constant. So the feedback effect $\sigma_{0}=\sigma_{0}(n)$ can be ignored in our case.

Equation (2) is a quasi-linear partial differential equation. Its characteristic equation and corresponding relations are:

$$
\begin{gathered}
\frac{\mathrm{d} c}{\mathrm{~d} t}=A\left(t, c, \sigma_{0}(n)\right) \\
\frac{\mathrm{d} n}{\mathrm{~d} t}=n_{\mathrm{N}}\left(t, c, \sigma_{0}(n)\right)-n\left(t, c, \sigma_{0}(n)\right) \frac{\partial n}{\partial c}
\end{gathered}
$$

respectively. Then, successive integration in the $(t, c)$ plane would provide a solution to eqn (2).

As a matter of fact, the general solution can be simplified in practical cases. We examine the special condition relevant to our experiment.

Curran et al. (1987) have found that the nucleation rate of total microcracks in planar impact tests is time independent. Shen et al. (1992) confirmed this assessment with the $\mathrm{Al}$ alloy used in this study. A series of planar impact tests with $c a .100$ ns loading duration was performed and the nucleation rate $n_{\mathrm{N}}$ for the $\mathrm{Al}$ alloy was approximated as a timeindependent product of two functions, both are single variable functions (Bai et al., 1992),

$$
n_{\mathrm{N}}\left(t, c, \sigma_{0}\right)=g\left(\sigma_{0}\right) f(c)
$$

Within the range of loading duration, in the "frozen in" tests, stress is nearly held constant. The stress-dependent function has been found to be either linear or exponential. Detailed discussions can be found in Curran et al. (1987), Shen et al. (1992) and Bai et al. (1992). 
Apart from a conslant or parametric stress, if the growth rate $A$ is also time-independent, the characteristics (5) and (6) can be integrated explicitly (Ke et al., 1990) as

$$
n\left(t, c, \sigma_{0}\right)=\frac{1}{A} \int_{c}^{c} n_{\mathrm{N}}\left(c^{\prime}, \sigma_{0}\right) \mathrm{d} c^{\prime}
$$

where, $\xi$ is defined by

$$
t=\int_{\xi}^{c} \frac{\mathrm{d} c^{\prime}}{A\left(c^{\prime}, \sigma_{0}\right)} .
$$

On the other hand, provided the nucleation law is known and the evolving distribution of microcracks $n\left(t, c, \sigma_{0}\right)$ can be recorded experimentally, we can derive the average growth rate $A$ inversely, in the light of eqn (2) and some proper boundary conditions. In the present study, we assumed the boundary condition as

$$
n\left(t, c, \sigma_{0}\right) \rightarrow 0 \quad \text { when } c \rightarrow 0
$$

since the measurement of microcracks, using scanning microscopy, showed that the number density distribution $n\left(t, c, \sigma_{0}\right)$ tends to zero when the size $c$ of visible microcracks is less than a micrometre. Thus

$$
A\left(t, c, \sigma_{0}\right)=\frac{1}{n} \int_{0}^{c}\left(n_{\mathrm{N}}-\frac{\partial n}{\partial t}\right) \mathrm{d} c .
$$

Because the remote loading is nearly held constant in the planar impact loading, the growth of a specified microcrack, i.e. a microcrack with specified current size $c$ and original size $c_{0}$, should not be different at different evolving times, i.e. the growth rate of microcracks should be explicitly time-independent. However, as shown in Section 4, the average growth rate $A$ obtained from the experiments did demonstrate time-dependence. In fact, the time-dependence of the average growth rate $A$ comes from the evolving distribution of microcracks. Hence, from a physical point of view, we introduce another growth rate of microcracks, $V$, which is dependent upon the current size of microcracks $c$, as well as its original size $c_{0}$, as most researchers suggested [see Curran et al. (1987)]. Therefore, the average growth rate $A$ is defined as

$$
A\left(t, c, \sigma_{0}\right)=\frac{\int_{\zeta}^{c} V\left(c, c_{0}, \sigma_{0}\right) n_{0}\left(t, c, c_{0}, \sigma_{0}\right) \mathrm{d} c_{0}}{\int_{\zeta}^{c} n_{0}\left(t, c, c_{0}, \sigma_{0}\right) \mathrm{d} c_{0}}
$$

where $V$ is the growth rate of microcracks with current size $c$ and original size $c_{0}$. Clearly $V$ should be independent of time $t . \zeta$ is the minimum nucleation size of all microcracks with size $c$ at time $t$. In eqn (11), $n_{0}$ represents the number density of microcracks in twodimensional phase space $\left(c, c_{0}\right)$. i.e. $n_{0}\left(t, c, c_{0}, \sigma_{0}\right) \mathrm{d} c \mathrm{~d} c_{0}$ is the number of microcracks with sizes $c \rightarrow c+\mathrm{d} c$ and nucleation sizes $c_{0} \rightarrow c_{0}+\mathrm{d} c_{0}$, so that, the sum of all cracks with sizes $c$ at time $t$ but with different nucleation sizes from minimum size $\zeta$ up to $c$ is equal to 


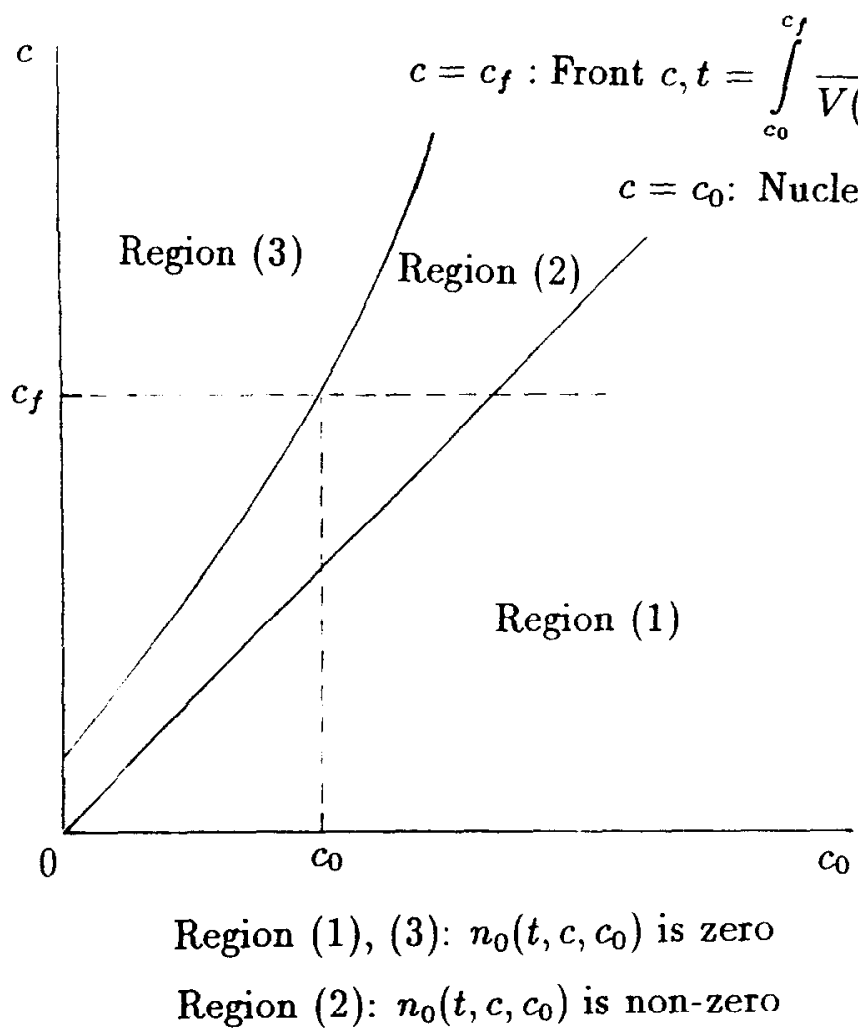

Fig. 2. Schematic region of $n_{0}\left(t, c, c_{0}\right)$.

$$
n\left(t, c, \sigma_{0}\right)=\int_{\zeta}^{c} n_{0}\left(t, c, c_{0}, \sigma_{0}\right) \mathrm{d} c_{0}
$$

In accord with the general formulation of microdamage evolution (1), $n_{0}$ should satisfy the following equation in an ideal system of microdamage,

$$
\frac{\partial n_{0}}{\partial t}+\frac{\partial\left(n_{0} V\right)}{\partial c}=n_{0, N}
$$

where $n_{0, \mathrm{~N}}$ is the nucleation rate in the two-dimensional phase space. The relationship between $n_{0, \mathrm{~N}}$ and $n_{\mathrm{N}}$ will be shown later. In particular, because the initial size $c_{0}$ remains invariant with time, $\partial\left(n_{0} V\right) / \partial c$ becomes the unique flux term in the equation.

Next, based upon the above results, we will solve eqn (12) and obtain the growth rate of microcracks $V$ from average growth rate $A$ by making use of eqn (11).

Now, we should examine the solution region of eqn (12) in the two-dimensional phase space (Fig. 2). In fact, the non-zero solution $n_{0}\left(t, c, c_{0}, \sigma_{0}\right)$ holds in the following region only

$$
n_{0}\left(t, c, c_{0}, \sigma_{0}\right)>0 \text { when } c_{0} \leqslant c \leqslant c_{\mathrm{f}}=c_{\mathrm{f}}\left(t, c_{0}, \sigma_{0}\right) \text {. }
$$

The requirement $c \geqslant c_{0}$ is quite trivial, because all current sizes of microcracks must be equal to or greater than their nucleation size $c_{0}$ due to the irreversibility of cracking. $c_{\mathrm{f}}=c\left(t, c_{0}, \sigma_{0}\right)$, the front of the number density of microcracks in the $c-c_{0}$ diagram is defined by 


$$
t=\int_{c_{0}}^{c_{f}} \frac{\mathrm{d} c^{\prime}}{V\left(c^{\prime}, c_{0}, \sigma_{0}\right)}
$$

or, $\zeta=\zeta\left(t, c, \sigma_{0}\right)$ is defined by

$$
t=\int_{\zeta}^{c} \frac{\mathrm{d} c^{\prime}}{V\left(c^{\prime}, \zeta, \sigma_{0}\right)} .
$$

Obviously, the above equations illustrate that the nucleated cracks with size $c_{0}$ will grow up to the size $c_{\mathrm{f}}$ at the growth rate $V$ after evolving time $t$; or, the cracks with current sizes $c$ at time $t$ come from the nucleated cracks with the nucleation size $\zeta$ at initial time. Moreover, the nucleation of microcracks can occur on $c=c_{0}$ only. Hence, the nucleation rate $n_{0, \mathrm{~N}}$ in two-dimensional phase space should be a $\delta$ function, satisfying the relation

$$
\int_{0}^{\infty} n_{0, N}\left(c, c_{0}, \sigma_{0}\right) \mathrm{d} c_{0}=n_{\mathrm{N}}\left(c, \sigma_{0}\right)
$$

so that,

$$
n_{0, N}\left(c, c_{0}, \sigma_{0}\right)=n_{\mathrm{N}}\left(c_{0}, \sigma_{0}\right) \delta\left(c-c_{0}\right)
$$

where $\delta$ is the Dirac- $\delta$ function with the inverse dimension that its argument has, i.e. $\left[\delta\left(c-c_{0}\right)\right]=1 /\left[\left(c-c_{0}\right)\right]=L^{-1}$, where [ ] denotes the dimension and $L$ is the dimension of length.

Substituting eqn (15) into eqn (12) and following the same procedure solving eqn (2) with parametric $\sigma_{0}$, we can easily derive the solution to eqn (12)

$$
n_{0}\left(t, c, c_{0}, \sigma_{0}\right)= \begin{cases}0, & \text { for } t<\int_{c_{0}}^{c} \frac{\mathrm{d} c^{\prime}}{V\left(c^{\prime}, c_{0}, \sigma_{0}\right)} \\ \frac{n_{\mathrm{N}}\left(c_{0}, \sigma_{0}\right)}{V\left(c, c_{0}, \sigma_{0}\right)} & \text { for } t \geqslant \int_{c_{0}}^{c} \frac{\mathrm{d} c^{\prime}}{V\left(c^{\prime}, c_{0}, \sigma_{0}\right)} .\end{cases}
$$

The solution (16) can also be deduced and interpreted as follows. Since there is no flux of number density of microcracks in the $c_{0}$ direction (Fig 2) for all microcracks, their nucleation size $c_{0}$ cannot be subjected to any variation with time. Hence, for a specified microcrack, i.e. a fixed $c_{0}$, the current size $c$ depends upon time as

$$
\Delta c=V\left(c, c_{0}, \sigma_{0}\right) \Delta t \quad \text { when } c_{0} \leqslant c \leqslant c_{\mathrm{f}}
$$

On the other hand, in the light of the nucleation and growth laws, and the property of the $\delta$ function, the flux of number density in microcracks should be expressed in the following way. All microcracks in the domain $\Delta c_{0} \Delta c$ come from the nucleation in $\Delta c_{0}$.

$$
n_{0}\left(t, c, c_{0}, \sigma_{0}\right) \Delta c \Delta c_{0}=n_{0, N}\left(c, c_{0}, \sigma_{0}\right) \Delta c \Delta c_{0} \Delta t=n_{\mathrm{N}}\left(c_{0}, \sigma_{0}\right) \Delta c_{0} \Delta t
$$

Substitution of eqn (17) into (18) gives

$$
n_{0}\left(t, c, c_{0}, \sigma_{0}\right) V\left(c, c_{0}, \sigma_{0}\right)=n_{\mathrm{N}}\left(c_{0}, \sigma_{0}\right) \quad \text { when } c_{0} \leqslant c \leqslant c_{\mathrm{f}} .
$$

This is the solution (16). From the above discussion, the implication of solution (16) becomes more clear. Firstly, for a prescribed point in the two-dimensional phase space $(c$, $c_{0}$ ), the number density $n_{0}$ remains constant with time owing to the time independent 
nucleation and the deterministic law of extension. Secondly, $\partial\left(n_{0} V\right) / \partial c=\partial n_{\mathrm{N}} / \partial c=0$ when $c_{0} \leqslant c \leqslant c_{\mathrm{f}}$, i.e. the flux is independent of current size $c$. Incidentally, a matter concerning the notification of the front should be emphasized. For specified $t$ and $c$, the front should be expressed in the second way of eqn (13b).

So the complete form of eqn (11) should be

$$
\begin{gathered}
A\left(t, c, \sigma_{0}\right)=\frac{\int_{\zeta}^{c} V\left(c, c_{0}, \sigma_{0}\right) n_{0}\left(t, c, c_{0}, \sigma_{0}\right) \mathrm{d} c_{0}}{n\left(t, c, \sigma_{0}\right)} \\
n=\int_{\zeta}^{c} n_{0}\left(t, c, c_{0}, \sigma_{0}\right) \mathrm{d} c_{0}=\int_{\zeta}^{c} \frac{n_{\mathrm{N}}\left(c_{0}, \sigma_{0}\right)}{V\left(c, c_{0}, \sigma_{0}\right)} \mathrm{d} c_{0} .
\end{gathered}
$$

Noticeably, eqn (20) represents the evolution of $n$ in terms of $n_{\mathrm{N}}$ and $V$.

Additionally, substitution of eqn (18) into (19) leads to a solution similar to our previous solution (8), in one-dimensional space but with time-dependent $A$.

$$
n\left(t, c, \sigma_{0}\right)=\frac{1}{A\left(t, c, \sigma_{0}\right)} \int_{\zeta}^{c} n_{\mathrm{N}}\left(c_{0}, \sigma_{0}\right) \mathrm{d} c_{0}
$$

Above all, provided the evolution $n\left(t, c, \sigma_{0}\right)$ and the nucleating rates $n_{\mathrm{N}}\left(c_{0}, \sigma_{0}\right)$ are known and the average extension rate $A\left(t, c, \sigma_{0}\right)$ is deduced from eqn $(10)$, a relation between $t, c$ and $\zeta$ can be established in terms of eqn (21) and eventually the physical growth rate $V$ can be derived as

$$
V=\left(\frac{\partial c}{\partial t}\right)_{\zeta}
$$

To summarize this section, we stress the two definitions of microcracks growth rate, $A$ and $V . A$ is the average growth rate of all microcracks with the same current size $c$, regardless of their original sizes $c_{0}$, whereas $V$ is the growth rate of microcracks with the same current size $c$ as well as original size $c_{0}$ but these microcracks may encounter possible fluctuations owing to microstructures. So physically, growth rate $V$ is much more meaningful. We shall discuss this matter in detail in Section 5.

\section{EXPERIMENTAL DESIGN AND DATA PROCESSING}

How did we get the evolution data of microdamage inside materials under impact loading? As mentioned in the Introduction, following Curran et al. (1987), we performed "frozen in" experiments with a light gas gun, but adopted some significant modifications. Curran et al. (1987) have described the "frozen in" tests in detail. The test is one in which known stress levels are applied "instantly", held constant for a known duration and then "instantly" removed; stress amplitudes and duration can be varied independently. In this way, microcracks can be frozen in at various levels of damage development. Then, metallographic examination of sectioned samples that have been exposed to the known stress and duration would provide necessary kinetics data. Our modification is mainly on the design of the flyer plate, in order to reveal microcrack kinetics at various stages of development under exactly identical stress levels in one shot and thus with less testing error. By taking advantage of the $101 \mathrm{~mm}$ bore of our gun, we designed a flyer with three of four blind holes of different depths on its free surface and then created tensile stresses of the 


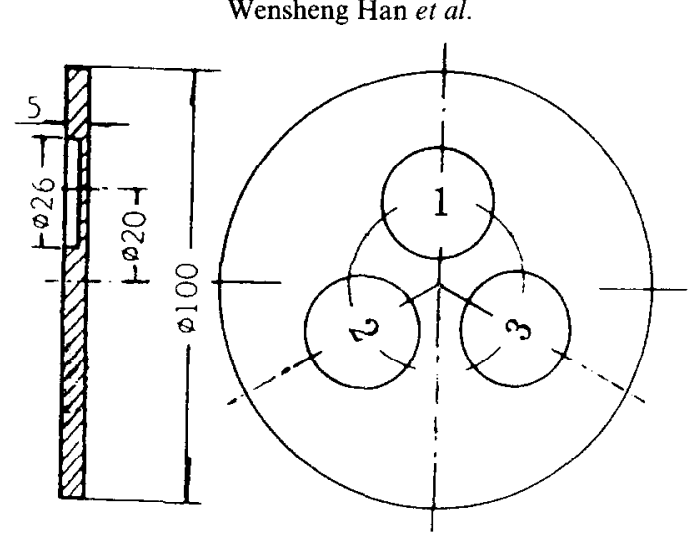

Fig. 3. Specially designed and machined flyer.

same amplitude $\sigma_{0}$, but for different tensile durations $t_{i}$ (Zhao et al., 1991) (Fig. 3). So in one shot, several different evolution stages of microdamage were frozen in, at different positions of a target plate. After planar impact, the target was recovered softly and sectioned at different positions corresponding to the holes of the flyer. The microcracks on sectional surfaces were counted and measured by an image analysis system

The counting of microcrack numbers demonstrates unavoidable fluctuations, even though the counting was made in tens viewfields. So, it is necessary to smooth the data in order to have a representative size distribution. Based on the sectional distributions of microcracks at different times $t_{i}$, a continuous sectional distribution of microcracks was deduced with Lagrangian interpolation,

$$
m\left(t, c^{\prime}, \sigma_{0}\right)=\sum_{k=0}^{3} \prod_{i=0, i \neq k}^{3}\left(\frac{t-t_{i}}{t_{k}-t_{i}}\right) m\left(t_{i}, c^{\prime}, \sigma_{0}\right) .
$$

The transformation from a sectional distribution $m\left(t, c^{\prime}, \sigma_{0}\right)$ to volumetric distributions $n\left(t, c, \sigma_{0}\right)$ was performed by making use of the following integral equation (Bai et al., 1993), see Appendix A.

$$
\frac{m\left(t, c^{\prime}, \sigma_{0}\right)}{c^{\prime}}=\int_{c^{\prime}}^{\infty} n\left(t, c, \sigma_{0}\right) \frac{\mathrm{d} c}{\sqrt{c^{2}-c^{\prime 2}}}
$$

and its solution (Appendix B)

$$
n\left(t, c, \sigma_{0}\right)=\frac{2}{\pi} \int_{c}^{\infty} \frac{c}{\sqrt{c^{\prime 2}-c^{2}}}\left(-\frac{1}{c^{\prime}} \frac{\mathrm{d} m\left(t, c^{\prime}, \sigma_{0}\right)}{\mathrm{d} c^{\prime}}+\frac{m\left(t, c^{\prime}, \sigma_{0}\right)}{c^{\prime}}\right) \mathrm{d} c^{\prime} .
$$

Therefore, the evolution data of microcracks $n\left(t, c_{0}, \sigma_{0}\right)$ were obtained from experimental measurements. The nucleation rate of microcracks $n_{\mathrm{N}}\left(c_{0}, \sigma_{0}\right)$ can be expressed as in eqn $(7)$ for the aluminium alloy. Then, the substitution of the evolution data of microcracks $n\left(t, c, \sigma_{0}\right)$ and the nucleation rate $n_{\mathrm{N}}\left(c_{0}, \sigma_{0}\right)$ into eqn $(10)$, would give the average growth rate $A\left(t, c, \sigma_{0}\right)$. Finally, the known functions $n\left(t, c, \sigma_{0}\right), A\left(t, c, \sigma_{0}\right)$ and $n_{\mathrm{N}}\left(c_{0}, \sigma_{0}\right)$, and eqn (21) lead to the relation between $t, c$ and $\zeta$, and the growth rate $V$ can be derived from eqn (22).

\section{CASE STUDY}

As a case study, the mentioned aluminium alloy subjected to planar impact was investigated. Both flyer and target were taken from the same Al alloy plate. Before testing, both flyer and target were polished carefully and adjusted to be parallel in order to guarantee normal impact and designed loading duration. All sectional surfaces were ground and 
Table 1. Specification of the test and relevant samples

\begin{tabular}{lccc}
\hline No. of blind holes & 1 & 2 & 3 \\
\hline Thickness of bottom of blind hole $(\mathrm{mm})$ & 0.642 & 1.361 & 2.258 \\
Speed of flyer $(\mathrm{m} / \mathrm{s})$ & & 172 & \\
Tensile stress (MPa) & 0.26 & 1470 & 0.50 \\
Duration of tensile stress $(\mu \mathrm{s})$ & 324 & 454 & 0.80 \\
Number of microcracks $\left(\mathrm{no} / \mathrm{mm}^{2}\right)$ & & 722 \\
\hline
\end{tabular}

polished with an Abramin automatic grinding and polishing machine. Then, with a Quantum 520 image analysis system, damaged regions on the surfaces were examined. For each sample, microcracks in about $10-20$ viewfields of $0.025 \mathrm{~mm}^{2}$ each were counted and summed to reduce fluctuations resulting from mesoscopic inhomogeneities. Figure 3 shows the design and dimensions of the flyer used in the case study. Table 1 lists specifications of the test and relevant samples. Figure 4 shows the sequential pictures of microcracks on sectional surfaces of samples $(<0.1 \mu \mathrm{s}, 0.26 \mu \mathrm{s}, 0.5 \mu \mathrm{s}$ and $0.8 \mu \mathrm{s}$, respectively).

To estimate possible errors involved in measurements of microcracks in the test, some preliminary measurements were made. Firstly, the transverse isotropy and homogeneity of the aluminium alloy plate were examined. On the plate surface grains were found to be roughly equiaxial, with ellipticity $(\sim 0.91)$. Whilst the ellipticity of grains on two sectioned surfaces perpendicular to each other are both 1.8 (Han, 1993). Then, from an arbitrary point on the plate surface we drew a straight line and counted the number of grains the line passed. The measurements showed that the grain number is independent of orientations of the straight line with variation less than $5 \%$ (Ling and Bai, 1994). These results justify statistical equivalence of measurements on different sectioned surfaces.

Then, possible sources of scatter and errors were estimated. The scatter of numbers of second phase particles measured on different sectional surfaces is about 5.2\% (Han, 1993), whereas numbers of microcracks in tested samples of different shots with similar loading conditions have greater variation, up to $10-20 \%$, even to $40 \%$. Nevertheless, statistical features of the obtained data are similar (Luo, 1988). Finally, the variation from sectional to volumetrical transformation was examined numerically. By taking parameters similar to our tests i.e. the total microdamage on the sectional surface is $10^{3} / \mathrm{mm}^{2}$ and the typical size of microdamage is $10 \mu \mathrm{m}$, the variation involved in the transformation (25) is $3 \%$ (Bai et al., 1993). Thus, we thought that our experimental data were representative.

Before going further, we should also examine the scatter of obtained data in the case study. For each sample, e.g. that of $0.2 \mu \mathrm{s}$, we have $36 \times 24,663 \mu \mathrm{m}^{2} \approx 0.88 \mathrm{~mm}^{2}$ viewfields. The deduced rate of nucleation are also listed in Table 1 . The least square fitting gives the nucleation rate of $928 / \mathrm{mm}^{2} \mu \mathrm{s}$. So, except for an overshoot at the beginning, the nucleation rate of microcracks seems to be time-independent in the time range up to $0.8 \mu \mathrm{s}$. This qualitatively agrees with simulations, in which just before EIC, only very few coalescences occur. Therefore, we thought that the model of ideal microcracks, i.e. negligible coalescence and annihilation, works in the case study.

Figure 5 shows the sectional number density distributions of microcracks $m\left(t_{i}, c^{\prime}, \sigma_{0}\right)$ appearing at different time intervals. The Fourier approximation technique is applied to eliminate fluctuations. Figure 6 shows the smoothed number density distributions $m\left(t_{i}, c^{\prime}, \sigma_{0}\right)$ and nucleation rate $m_{\mathrm{N}}\left(c^{\prime}, \sigma_{0}\right)$. Figure 7 shows the volumetric distribution $n\left(t_{i}, c, \sigma_{0}\right)$ and $n_{\mathrm{N}}\left(c_{0}, \sigma_{0}\right)$ completed in accord with the equation of transformation and its solution (24) and (25). Figure 8 demonstrates that the average growth rate of microcracks $A$ is dependent upon the size $c$ as well as time $t$ derived from eqn (10). This is not physically meaningful. Figure 9 shows the growth rate of microcracks $V$ versus the size $c$ and the nucleation size $c_{v}$ in our experimental range from $0.26 \mu$ s to $0.80 \mu \mathrm{s}$. Figure 10 provides the growth rate of microcracks $V$ as a function of $c_{0} / c$ in the same range of time, but unfortunately it was found that unlike the expressions derived by Mott (1948), Berry (1974) and others, the physical growth rate $V$ is not the unique fluctuation of $c_{0} / c$. Finally Fig. 11 shows that the data of growth rate $V$ collapse to a function of a single variable-incremental 


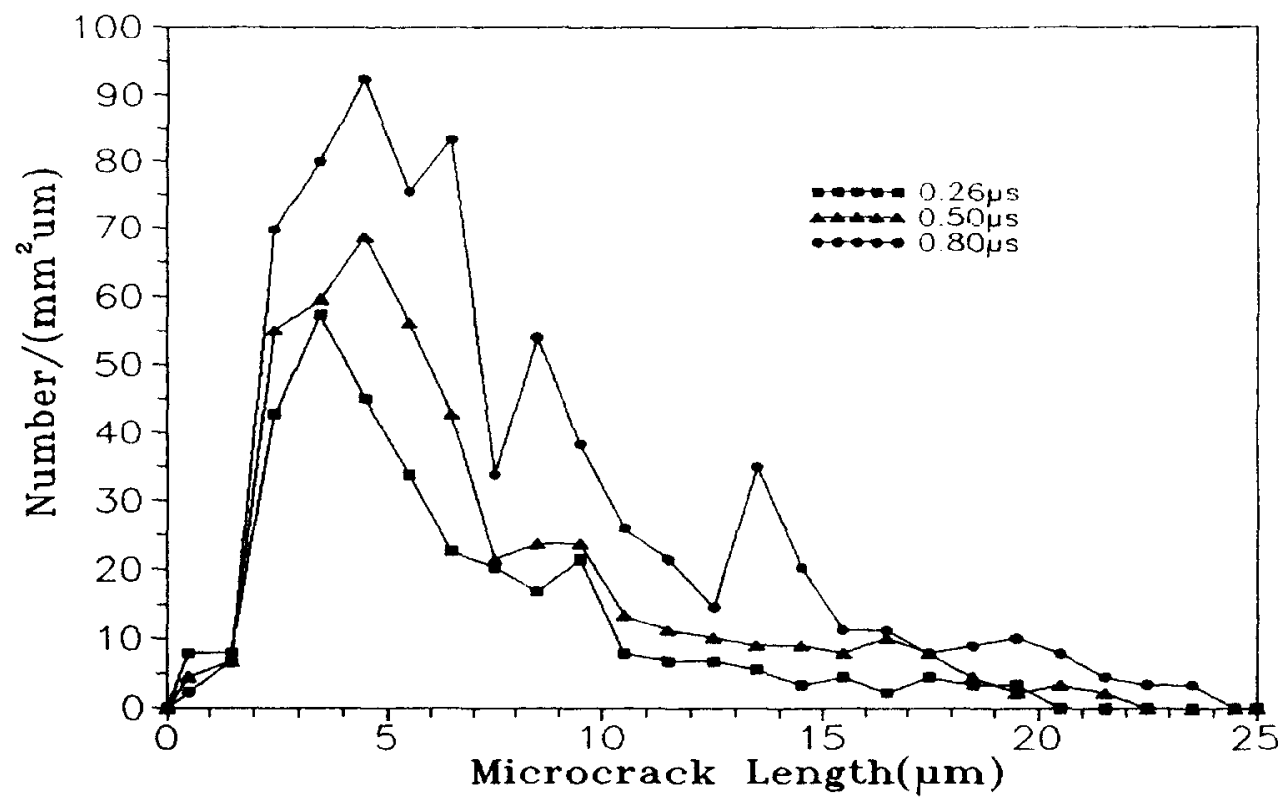

Fig. 5. Sectional number density distribution of microcracks $m\left(c^{\prime}, t_{i}\right)$.

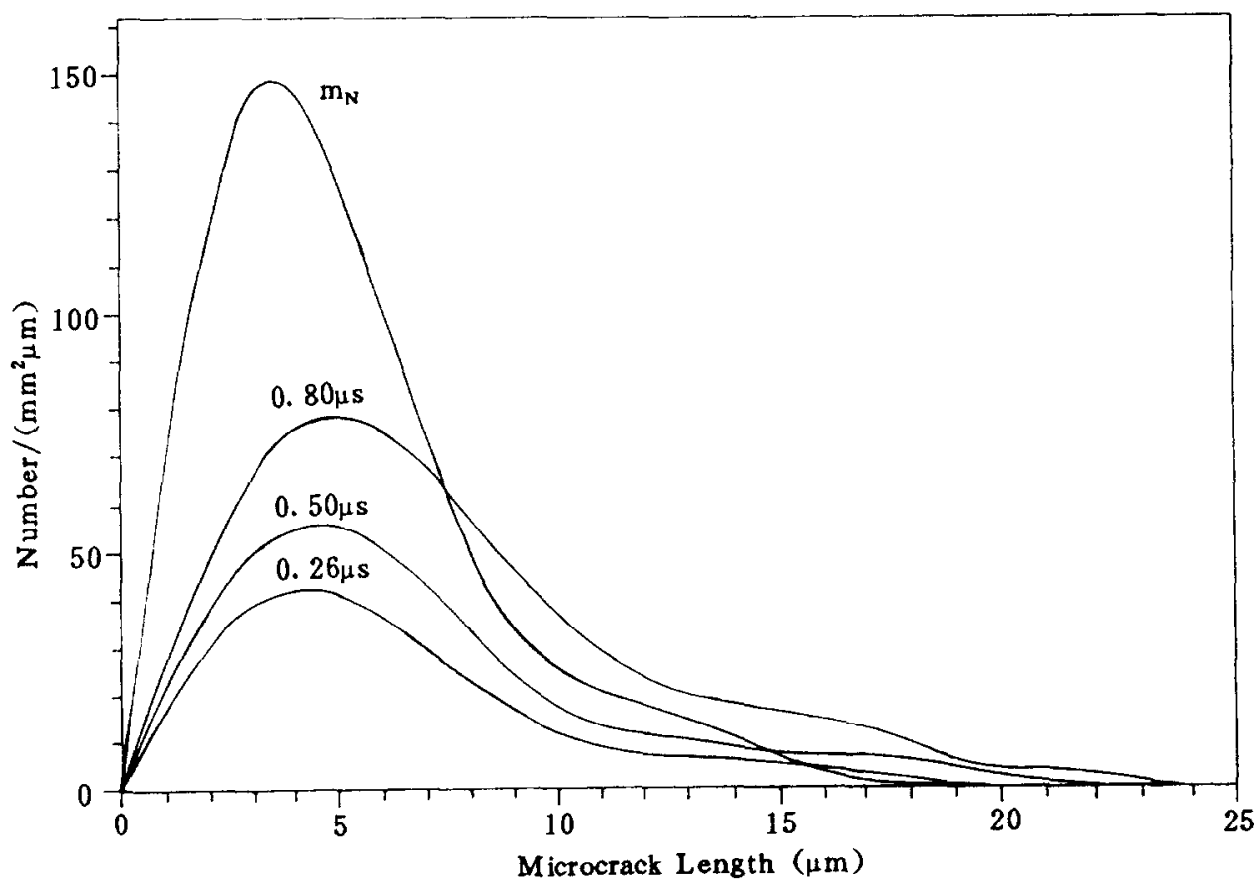

Fig. 6. Smoothed number density distribution $m\left(c^{\prime}, t_{i}\right)$ and nucleation rate $m_{\mathrm{N}}\left(c^{\prime}\right)$.

extension $\left(c-c_{0}\right)$. The formula of the growth rate of microcracks $V$ fitted to all available data was written as

$$
V\left(c, c_{0}\right)=V^{*}\left(\frac{c-c_{0}}{c_{0}}\right)^{0.775}
$$

where $c_{0}$ is the average nucleation size of microcracks, $V^{*}=8.1 \mu \mathrm{m} / \mu \mathrm{s}$. With this formula (26) and eqns (13) and (20), the evolution of the number density distribution $n\left(t, c, \sigma_{0}\right)$ was 


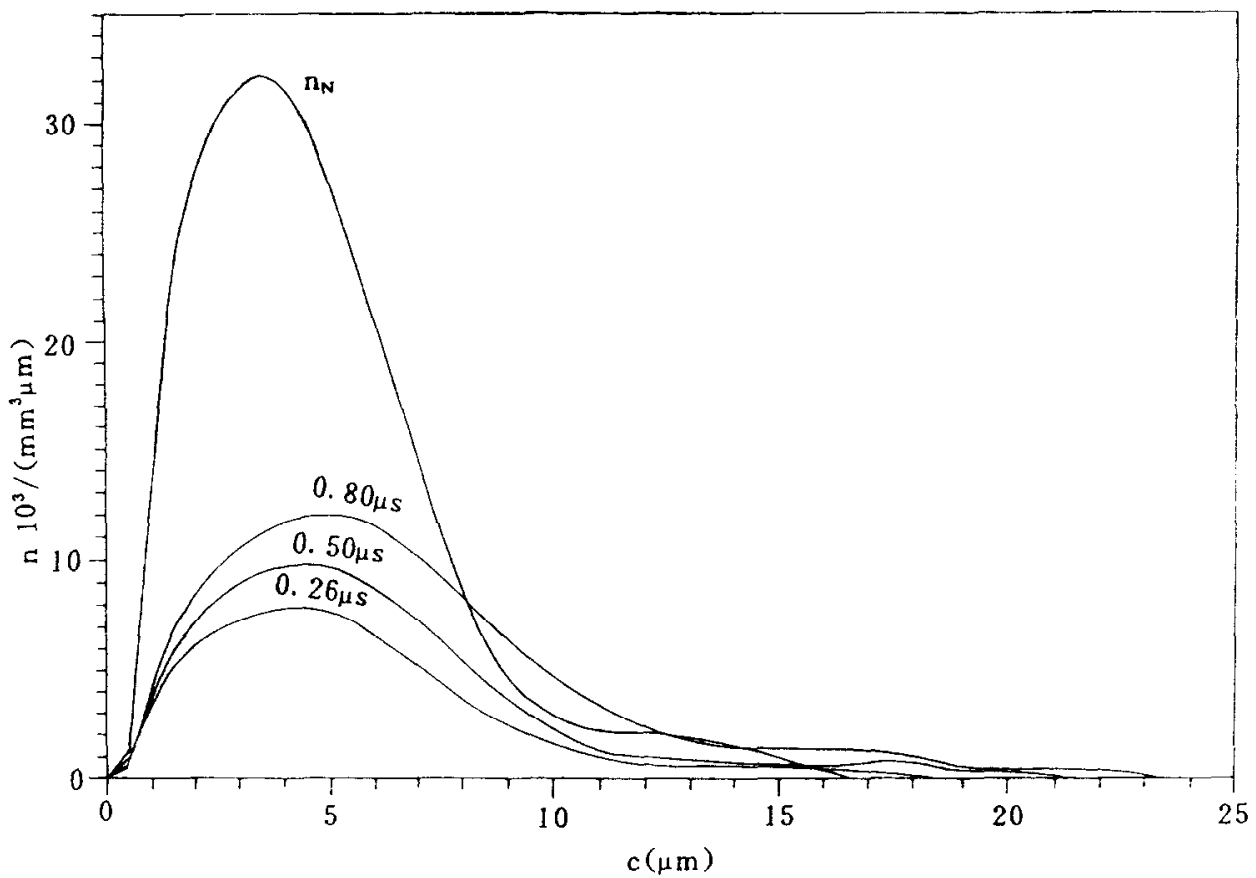

Fig. 7. Volumetric number density distribution $n\left(c, t_{i}\right)$ and nucleation rate $n_{\mathrm{N}}(c)$.

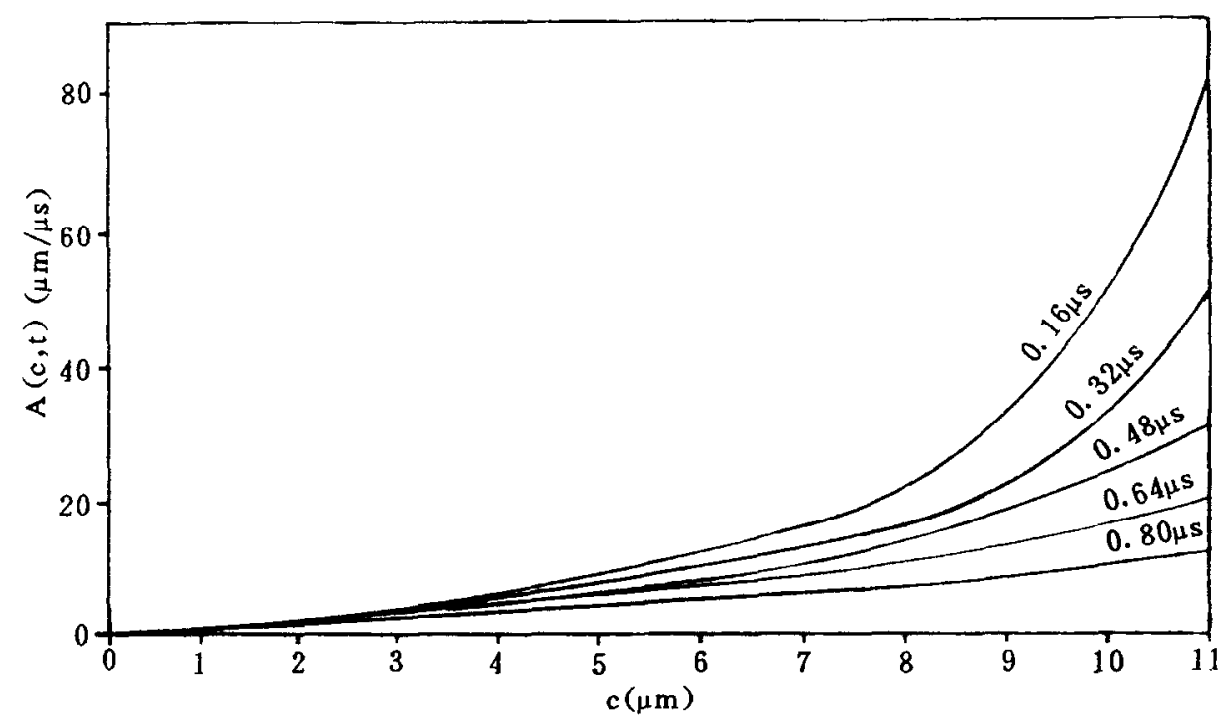

Fig. 8. Average growth rate of microcracks $\mathrm{A}$.

calculated again. Figure 12 shows the comparison of the calculated evolution of number density of microcracks and the experimental ones. The agreement is fairly good.

\section{DISCUSSION AND SUMMARY}

(1) When looking back to the proposed approach for the determination of growth rate of microcracks, one may ask whether the growth rate $V$ is meaningful in practice. To answer the question, let us start from the very beginning and examine the $i$-th individual microcracks with current size $c$ and initial size $c_{0}$, respectively, under remote stress $\sigma_{0}$. If we were tracking the microcrack with a microscope, we would obtain its growth rate 


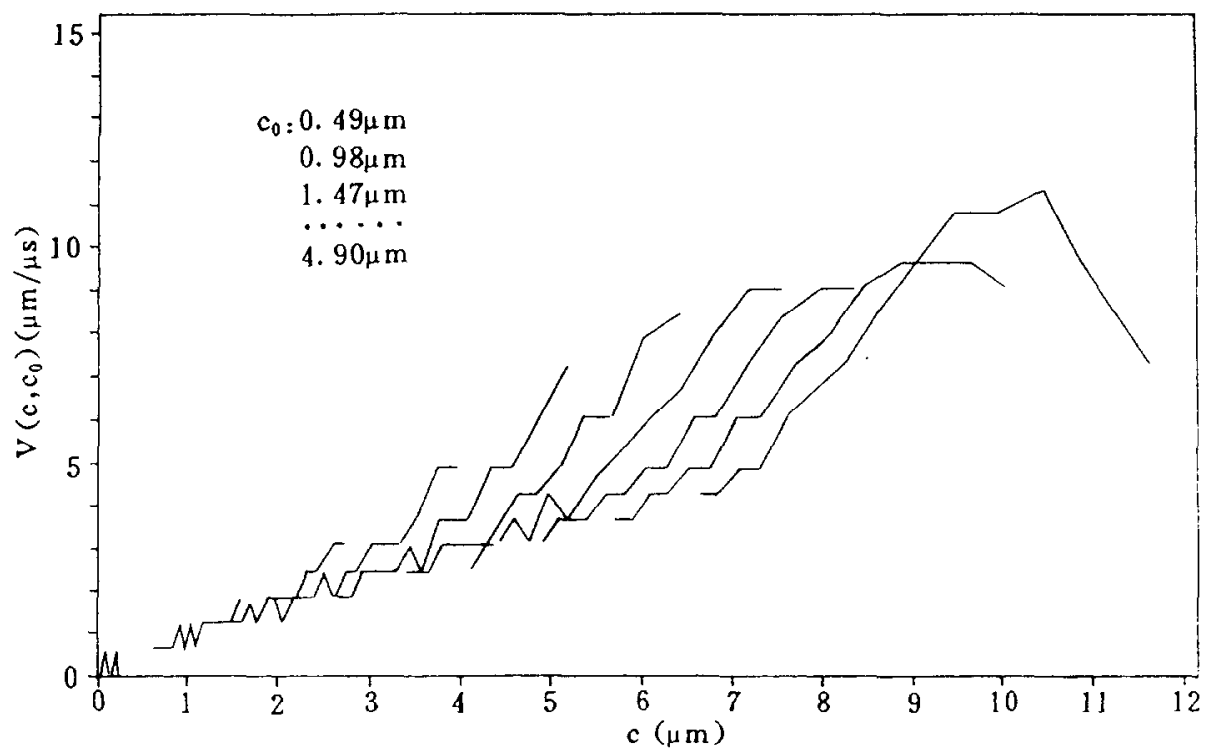

Fig. 9. The growth rate of microcracks $V$ versus the size $c$ and the nucleation size $c_{0}$ in the experimental range $(0.26 \mu \mathrm{s}-0.80 \mu \mathrm{s})$.

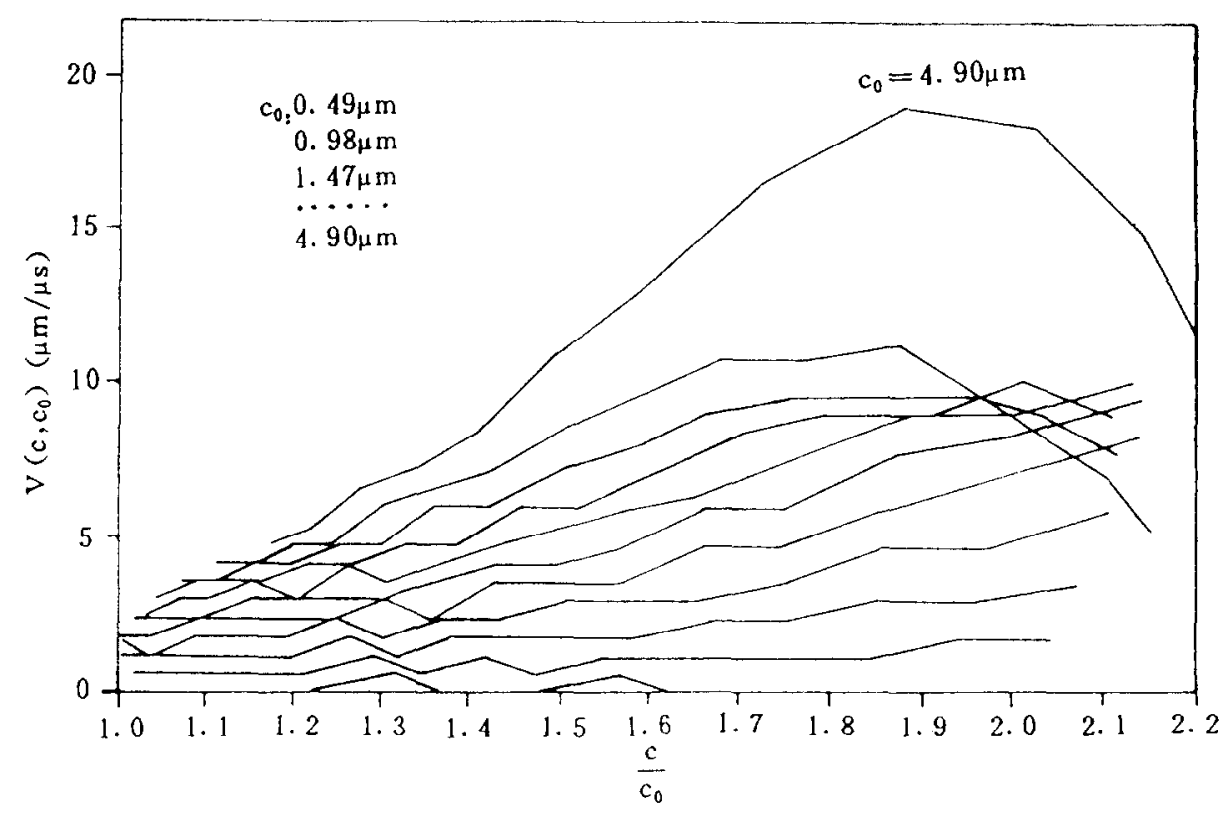

Fig. 10. The growth rate of microcracks $V^{r}$ versus $c_{0} / c$.

$v=v\left(i, c, c_{0}, \sigma_{0}\right)$, where $i$ denotes the $i$-th crack. Owing to the difference of the microstructure, such as grain boundaries, defects, etc. adjacent to the tip of the microcrack, $v$ would sustain some perturbations. So,

$$
v\left(i, c, c_{0}, \sigma_{0}\right)=V\left(c, c_{0}, \sigma_{0}\right)+\tilde{v_{i}}
$$

where

$$
V=\langle v\rangle=\sum_{i}^{n_{0}} v / n_{0}
$$




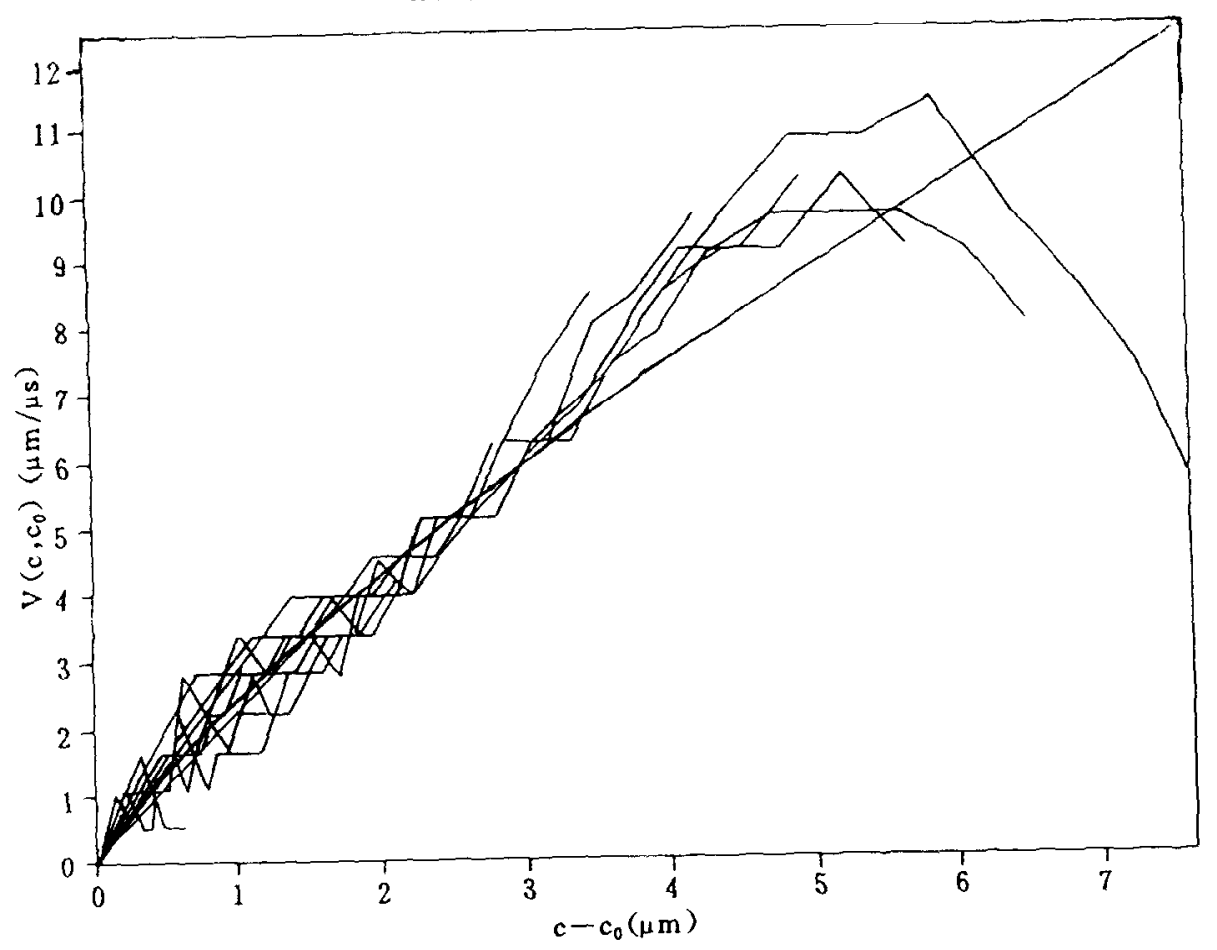

Fig. 11. The growth rate $V^{r}$ versus $\left(c-c_{0}\right)$ and a fitted line.

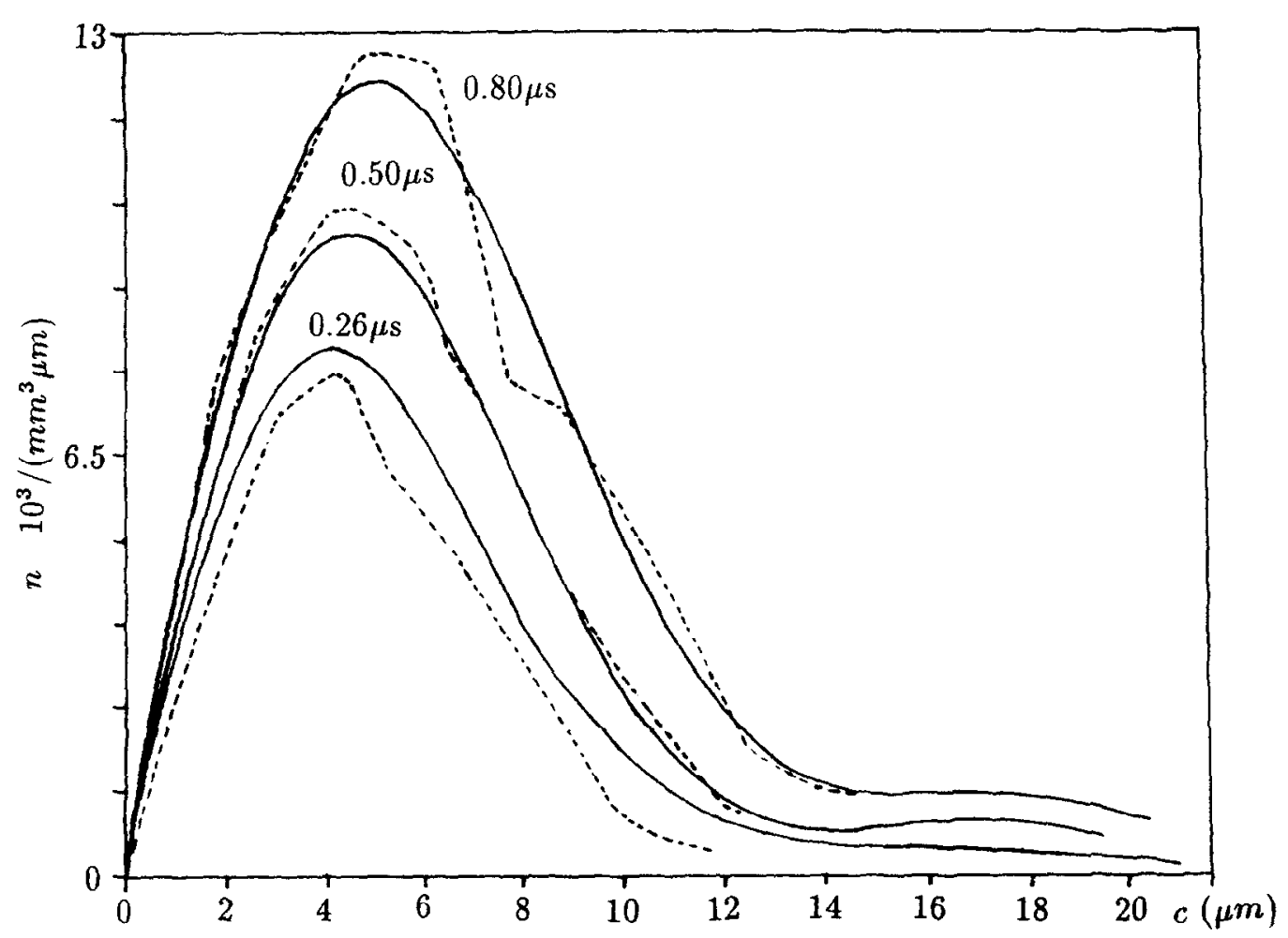

Fig. 12. Comparison of the calculated evolution of number density of microcracks and the experimental ones (dashed line is the calculated result).

and $\tilde{v_{\mathrm{i}}}$ is the perturbation. Clearly

$$
\sum_{i}^{n_{0}} \tilde{v}_{i} n_{0}=0
$$


Now let us investigate if the perturbation $\tilde{v_{i}}$ has any effect on the statistical evolution of microcracks. We may have the following relations,

$$
t_{i}=\int_{\zeta}^{c} \frac{\mathrm{d} c^{\prime}}{v\left(i, c^{\prime}, \zeta, \sigma_{0}\right)}
$$

However, this cannot affect our results (18) and (19), for example,

$$
A=\int_{\zeta}^{c}\left(\sum_{i}^{n_{0}} v_{i}\right) \mathrm{d} c_{0} / \int_{\zeta}^{c} n_{0} \mathrm{~d} c_{0}=\int_{\zeta}^{c}\left(V n_{0}+\sum_{i}^{n_{0}} \tilde{v}_{i}\right) \mathrm{d} c_{0} / \int_{\zeta}^{c} n_{0} \mathrm{~d} c_{0}=\int_{\zeta}^{c} V n_{0} \mathrm{~d} c_{0} / \int_{\zeta}^{c} n_{0} \mathrm{~d} c_{0} .
$$

In one word, it is the growth rate $V$ rather than the individual growth rate $v$ that governs the statistical evolution of microcracks. The latter, $v$, determined by tracking individual microcracks is less important in the evolution, except for the case when the microcrack has become the main crack.

(2) A number of models of growth of cracks and voids have been proposed as follows

$$
\begin{gathered}
\text { Plastic model (Rice and Tracey, 1969) } \dot{R}=\left(\frac{2}{3} \bar{\varepsilon}\right)^{1 / 2} 0.558 \sinh \left(\frac{\sqrt{3} \sigma_{0}}{2 \tau_{0}}\right) \sim R \\
\text { Viscous model (Curran et al., 1987) } \quad \dot{R}=T \frac{P_{s}-P_{n 0}}{4 \eta} R \sim R
\end{gathered}
$$

$$
\text { Cleavage model (Mott, 1948) } \dot{R}=V_{\mathrm{T}}\left(1-R^{*} / R\right)^{1 / 2} \sim\left(1-\frac{R^{*}}{R}\right)^{1 / 2}
$$

where $R$ is the current size of void or crack. Generally speaking, eqns (29) and (30) belong to the type of ductile energy-dissipation, whereas eqn (31) is brittle. The comparison of our experimental result (26) and the above three formulae indicates that the mechanism controlling the concerned case may be of ductile dissipation. Indeed, some of our impact tests with longer loading duration created such fracture surfaces that there are dimple networks around second phase particles. We also tried a linear fitting of our experimental data,

$$
V=K\left(\begin{array}{c}
c-c_{0} \\
c_{0}
\end{array}\right)
$$

After disregarding a few data of decreasing growth rate, although slightly arbitrarily, the fitting formula (32) gives $K=7.1 \mu \mathrm{m} / \mu \mathrm{s}^{-1}$. This seems quite reasonable (Fig. 13) and does imply viscous mechanics of microcrack growth in the Al alloy. After conducting their iterative procedure and computational simulation, Curran et al. (1987) have suggested that "for many structural materials, plastic flow at the microcrack tips prevents brittle growth behavior at high imposed tensile stresses, and instead a viscous growth law like that discussed above for ductile void is appropriate". Our results appear to justify this statement, but instead of the current size, the increment of microcracks appears in our results. The reason for this is still under study.

(3) Previously, we had introduced the correlation of the macroscopic measure of damage $D$ and the number density of microdamage $n$ (Bai et al., 1991). For example, 


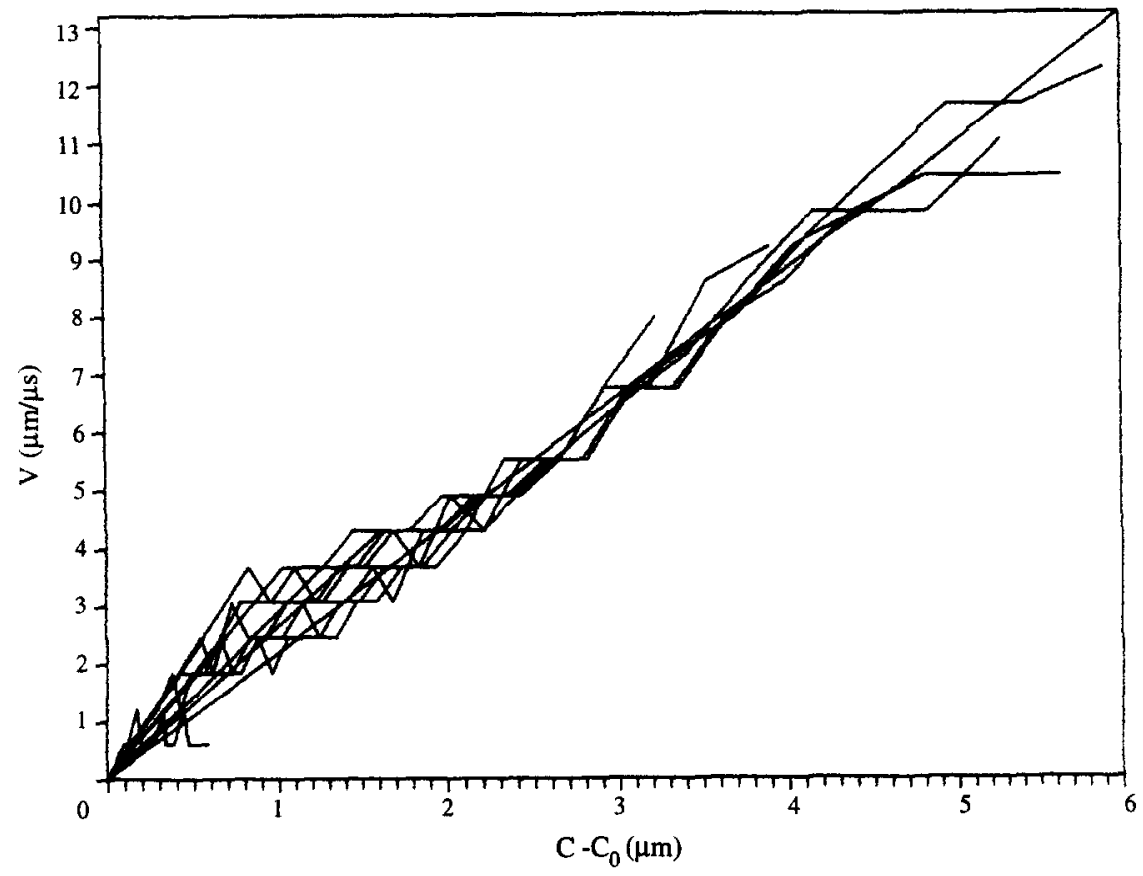

Fig. 13. The linear fitting of growth rate of microcrack disregarding a few decreasing dala.

$$
\begin{gathered}
D_{0}=\int_{0}^{\infty} n \mathrm{~d} c \\
D_{3}=\int_{0}^{\infty} n c^{3} \mathrm{~d} c \sim f
\end{gathered}
$$

$D_{3}$ is proportional to the volumetric fraction of microdamage, $f$, a macroscopic measure of damage. Substitution of the solution (20) into eqns (33) and (34), and exchange of integrals give

$$
\begin{aligned}
D_{0}\left(t, \sigma_{0}\right)= & \int_{0}^{\infty} n_{\mathrm{N}}\left(c_{0}, \sigma_{0}\right) \mathrm{d} c_{0} \int_{c_{0}}^{c_{f}} \frac{\mathrm{d} c}{V\left(c, c_{0}, \sigma_{0}\right)}=\int_{0}^{\infty} n_{\mathrm{N}}\left(c_{0}, \sigma_{0}\right) \mathrm{d} c_{0} \cdot t \\
& D_{3}\left(t, \sigma_{0}\right) \partial \int_{0}^{\infty} n_{\mathrm{N}}\left(c_{0}, \sigma_{0}\right) \mathrm{d} c_{0} \int_{c_{0}}^{c_{f}} \frac{c^{3} \mathrm{~d} c}{V\left(c, c_{0}, \sigma_{0}\right)} .
\end{aligned}
$$

Clearly, the evolution of number density $n$ plays the key part in the damage. Furthermore, it is an integration of combined nucleation and growth laws of microdamage. This implies that some fluctuations in the two mesoscopic dynamic processes can be smoothed in the macroscopic measure of damage. However, the singularity of the integrand $1 / V\left(c, c_{0}, \sigma_{0}\right)$ coming from the initial growth $V\left(c, c_{0}, \sigma_{0}\right)$ may become the most important mesoscopic effect on the macroscopic mechanical damage.

(4) In the case study of the paper, even for the sample subjected to the longest loading duration $(0.8 \mu \mathrm{s})$, the damage fraction is still not very large, $c a$. one tenth. So, the stress approximately holds constant, hence the model of ideal microcracks and corresponding solutions, like eqn (21), are valid. In fact, the model of ideal microcracks and eqns (2) and (12) can still work with mean field approximation. That is to say, the feedback effect $\sigma_{0}=\sigma_{0}[D(n)]$, due to interaction of microcracks can be included in the model of ideal microcracks. Details on this matter can be found in Bai et al. (1994c). This model has also been applied to microcracks occurring in fatigue tests. 
(5) Now we would like to summarize the present study as follows

- A new statistical formulation of microcracks in two-dimensional phase space $\left(c, c_{0}\right)$ was developed. The evolution of number density of ideal microcracks can be expressed by their mesodynamic laws of nucleation $n_{\mathrm{N}}$ and growth rate $V$ as in eqn (20).

- An cxperimental approach to the determination of the growth rate of microcracks directly from experimental data was proposed. A practical example of aluminium alloy subjected to submicrosecond stress was presented.

- The mechanism governing the growth of microcracks should be attributed to ductile dissipation. Also, the fracture surface demonstrates ductile dimples. The growth rate of microcracks in the aluminium alloy is about $10 \mathrm{~m} \mathrm{~s}^{-1}$, under a tensile stress of 1470 $\mathrm{MPa}$ and load duration of $0.26 \mu \mathrm{s}-0.80 \mu \mathrm{s}$.

Acknowledgements-The work was supported by the National Natural Science Foundation of China and the Chinese Academy of Sciences under special grant KM 85-33. The authors are grateful to Prof. Fujiu Ke and Ms Shuxia Chen for their help.

\section{REFERENCES}

Bai, Y. L., Ke, F. J. and Luo, L. M. (1988). Statistical modeling of damage evolution in spallation. Journal de Physique Collogue C3 49, 215-221.

Bai, Y. L., Ke, F. J. and Xia, M. F. (1991). Formulation of statistical evolution of microcracks in solids. Acta Mechanica Sinica 7, 59-66.

Bai, Y. L., Ke, F. J. and Xia, M. F. (1994a). Analysis and simulation of evolution induced catastrophe. Chinese Science Bulletin. 10, 155-158.

Bai, Y. L., Lu, C. S., Ke, F. J. and Xia, M. F. (1994b). Evolution induced catastrophe. Physics Letters A. 185, $196-200$.

Bai, Y., L., Han, W. S., Xia, M. F., Ke, F. J. and Lu, C. S. (1994c). Material instability due to evolution of microdamage. In Material Instabilities, Theory and Application (Edited by R. C. Batra and H. M. Zbib), pp. 111-116. ASME Winter Annual Meeting, Chicago.

Bai, Y. L., Xia, M. F., Ke, F. J., Guo, W. H. and Ling, Z. (1993). Statistical interpretation on the relationship between the volumetric and sectional size distributions of disordered mesostructures. Acta Physica Sinica 42, 353-359

Bai, Y. L., Ling, Z., Luo, L. M. and Ke, F. J. (1992). Initial development of microdamage under impact loading. Transactions of the ASME Journal of Applied Mechanics 59, 622-627.

Berry, J. P. (1960). Some kinetic considerations of the Griffith criteria for fracture. J. Mech. Phys. Solids. 8, 194.

Curran, D. R., Seaman, L. and Shockey, D. A. (1987). Dynamic failure of solids. Physics Reports $147,253$.

Fineberg, J., Gross, S. P., Marder, M. and Swinney, H. L. (1992). Physics Review B 10, 5146.

Freund, L. B. (1992). Lecture at the Institute of Mechanics. Beijing, China.

Gurson, A. L. (1975). Transactions of the ASME, H: Journal of Engineering Materials Technology $99,2$.

Han, W. S. (1993). Meso-mechanical laws governing spallation in an aluminium alloy. Ph.D. Thesis, Inst. Mech. CAS.

Ke, F. J., Bai, Y. L. and Xia, M. F. (1990). Evolution of ideal microcrack system. Science in China 33, $1447-1459$.

Ling, Z., and Bai, Y. L. (1994). Explosion and Shock Waves 14, 1.

Luo, L. M. (1988). An expcrimental study of nucleation processes and modeling of damage evolution in spallation in an Al alloy. M.Sc. thesis, Inst. Mech. CAS.

Mott, N. F. (1948). Engineering 165, 16.

Needleman, A. and Tvergaard, V. (1992). Applied Mechanics Review 45, 33

Prakash, V., Freund, L. B. and Clifton, R. J. (1992). Transactions Journal of Applied Mechanies 59, 356.

Rice, J. R. and Tracey, D. M. (1969). Journal of Mechanics and Physics of Solids. 17, 202.

Scheil, V. E. (1931). Anarg. Allg. Cham. 201, 259.

Scheil, V. E. (1935). Metallk. 27, 199.

Shen, L. T., Zhao, S. D., Bai, Y. L. and Luo, L. M. (1992). International Journal of Impact Engineering $12,9$.

Shioya (1993). In Proceedings of IITAM Symposium on Impact Dynamics, Beijing, China.

Underwood, E. E. (1970). Quantitative Stereology. Addison-Wesley, Reading, Mass.

Xia, M. F., Bai, Y. L. and Ke, F. J. (1994). Science in China, 37, 331.

Xing, S. X. (1986). Advances in Mechanics 16, 495.

Zhao, S. D., Ling, Z., Shen, L. T., Chen, S. X. and Bai, Y. L. (1991). In Proceedings of Shock Compression of Condensed Matter. Elsevier Science.

\section{APPENDIX A. THE DERIVATION OF EQN (24)}

The derivation of the fundamental integral equation governing the transformation of sectional observations of meso-structures to their volumetric size distribution is based upon the following assumptions:

(1) The concerned mesoscopic structures have the simplest configuration, i.e. spheres or parallel pennyshaped cracks.

(2) The spheres and penny-shaped cracks are homogeneously, randomly distributed in the materizl. 
It is worth noting that no limitation has been imposed on the form of size distribution. Firstly, we define the following quantities to describe their size distribution of these meso-structures.

$c$ : length scale of meso-structures, i.e. the true diameter of sphere of penny-shaped cracks

$c^{\prime}$ : apparent length scale of meso-structures on a sectional surface, i.e. the diameter of the apparent circle of sphere or the length of the apparent crack line of penny-shaped crack on sectional surface;

$n(c)$ : volumetric number density of the meso-structures. For instance, $n(c) \mathrm{d} c$ represents the number of spheres in unit physical volume and in the interval of sphere-diameter $c \rightarrow c+\mathrm{d} c$;

$m\left(c^{\prime}\right)$ : sectional number density of the meso-structures. For instance, $m\left(c^{\prime}\right) \mathrm{d} c^{\prime}$ represents the number of apparent circles of spheres, which are sectioned and shown themselves as circles with a diameter range of $c^{\prime} \rightarrow$ $c^{\prime}+\mathrm{d} c^{\prime}$ in the unit physical area on the sectional surface.

To determine the number of cracks or voids within a given size range, say from $c_{i}^{\prime}$ to $c_{i+1}^{\prime}$ and $c_{j}$ to $c_{j+1}$, we form the integrals :

$$
M_{1}\left(c^{\prime}\right)=\int_{c_{i}^{\prime}}^{c_{i-1}^{\prime}} m\left(c^{\prime}\right) \mathrm{d} c^{\prime}
$$

and

$$
N(c)=\int_{c_{j}}^{c_{j+1}} n(c) \mathrm{d} c .
$$

Thus, we see that the units for $m\left(c^{\prime}\right)$ and $n(c)$ are number per unit length ${ }^{3}$ and number per unit length ${ }^{4}$.

The basic idea of the derivation of the equation governing the transformation is due to previous works (Scheil, 1931, 1935; Underwood, 1970) and can be explained as follows. In the case of spheres, the observed number $m\left(c^{\prime}\right) \mathrm{d} c^{\prime}$ of circles on a sectioned surface in the interval of diameter $c^{\prime} \rightarrow c^{\prime}+\mathrm{d} c$ is attributed to the spheres, which have diameters greater than $c^{\prime}$ and are sectioned in the concerned volume. Furthermore, for an assigned sphere, its probability to be sectioned to become circles with diameter $c^{\prime}$ between 0 and $c$ can be determined according to equal probability of circles with the same increment in its height. In order to sketch the idea clearly, a penny-shaped crack and its sectional line are shown in Fig. Al. It can be verified that the case of spheres follows the same procedure, reasoning and result.

For a penny-shaped crack with diameter, $c=2 R$, its probability $p\left(c^{\prime}\right) \mathrm{d} c^{\prime}$ showing a crack line with a length in $c^{\prime} \rightarrow c^{\prime}+\mathrm{d} c^{\prime}$ on a sectional surface is

$$
p\left(c^{\prime}, c\right) \mathrm{d} c=-\frac{\mathrm{d} r}{R}=\frac{1}{c} \frac{c^{\prime} \mathrm{d} c^{\prime}}{\sqrt{c^{2}-c^{2}}}
$$

due to

$$
r^{2}=R^{2}-\left(\frac{c^{\prime}}{2}\right)^{2} \text { and } d r=-\frac{c^{\prime} \mathrm{d} c^{\prime}}{2 \sqrt{c^{2}-c^{\prime 2}}}
$$

If each crack is represented by its mass centre, then the number of cracks with diameters between $c$ and $c+\mathrm{d} c$ in a given volume $c \cdot l^{2}$, (Fig. A1) is
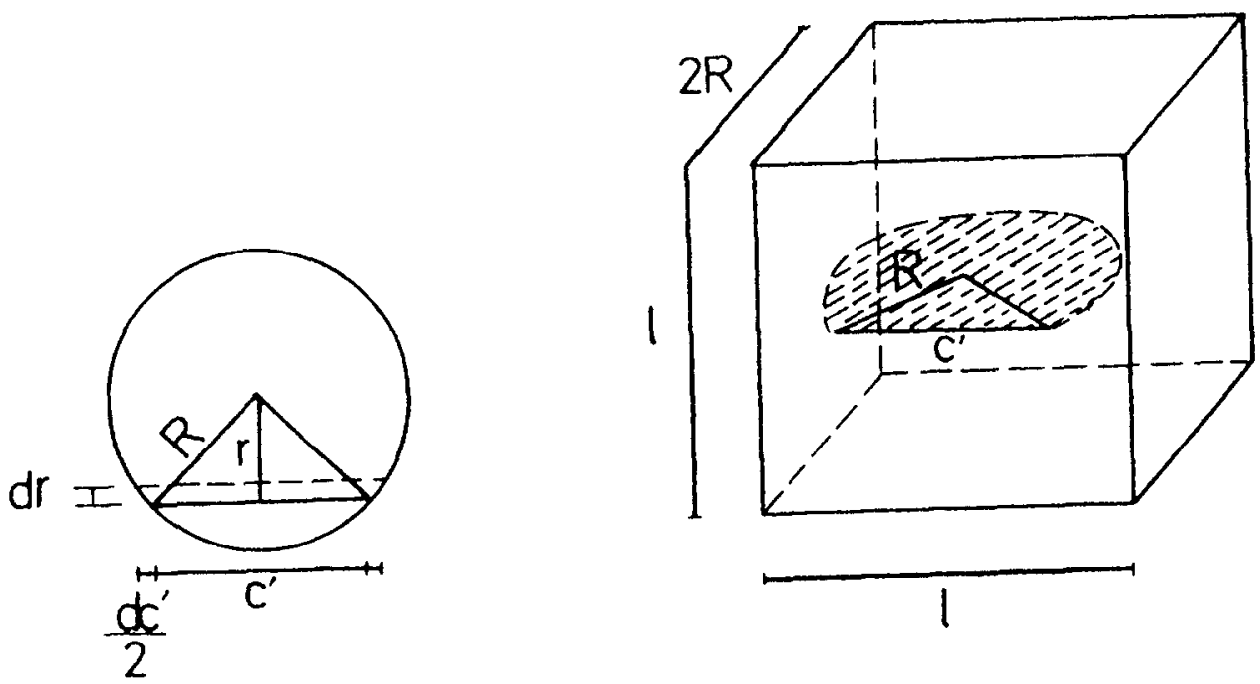

Fig. Al. A penny-shaped crack and its sectional line. 


$$
m\left(c^{\prime}\right) \mathrm{d} c^{\prime} \cdot l^{2}=\int_{i=c^{\prime}}^{\infty} n(c) \mathrm{d} c \cdot c \cdot l^{2} \cdot p\left(c^{\prime}, c\right) \mathrm{d} c^{\prime}=\int_{c=c^{\prime}}^{\infty} n(c) \mathrm{d} c \cdot c \cdot l^{2} \cdot \frac{1}{c} \frac{c^{\prime} \mathrm{d} c^{\prime}}{\sqrt{c^{2}-c^{\prime 2}}}
$$

The integral is due to the contribution made by all the penny-shaped cracks with diameter greater than $c^{\prime}$ and corresponding probability $p\left(c^{\prime}\right) \mathrm{d} c^{\prime}$. Now the governing integral equation can be written as follows

$$
\frac{m\left(c^{\prime}\right)}{c^{\prime}}=\int_{r}^{\infty} n(c) \frac{\mathrm{d} c}{\sqrt{c^{2}-c^{\prime 2}}}
$$

where $m\left(c^{\prime}\right)$ is the observed quantity on the sectional surface, and $n(c)$ is the unknown volumetric size distribution. Obviously, this is a first-kind Volterra's integral equation, but with infinite integral limit and singular kernel.

\section{APPENDIX B. THE DERIVATION OF EQN (25)}

Usually, the function $m\left(c^{\prime}\right)$ is generally normal in practice. Noticeably, the kernel $1 / \sqrt{c^{2}-c^{\prime 2}}$ is continuous and differential in the range $\left(c^{\prime}, \infty\right)$, but with $(-1 / 2)$ power singularity at lower integral limit $c=c^{\prime}$. This shows apparent similarity to the Abel's integral equation.

To solve the integral equation, we multiple eqn (A3) by $1 / \sqrt{c^{2}-z^{2}}$ and then integrate it with respect to $c^{\prime}$ between the limits $c^{\prime}=z$ and $c^{\prime}=\infty$ :

$$
\int_{z}^{\infty} m\left(c^{\prime}\right) \frac{\mathrm{d} c^{\prime}}{\sqrt{c^{\prime 2}-z^{2}}}=\int_{z}^{\infty} c^{\prime} \frac{\mathrm{d} c^{\prime}}{\sqrt{c^{\prime 2}-z^{2}}} \int_{c^{\prime}}^{\infty} n(c) \frac{\mathrm{d} c}{\sqrt{c^{2}-c^{\prime 2}}}
$$

where $z$ is an intermediate variable, an alternative representation of length scale, either $c$ or $c^{\prime}$. The integral region of the double integral in the right hand side of eqn $(\mathrm{B} 1)$ is the shaded angular domain in the $c-c$ plane, as shown in Fig. B1. When we exchange the order of the double integral, expression (B1) should be rewritten with correspondingly changed integral limits as follows

$$
\int_{z}^{\infty} \frac{m\left(c^{\prime}\right)}{\sqrt{c^{\prime 2}-z^{2}}} \mathrm{~d} c^{\prime}=\int_{z}^{\infty} n(c) \mathrm{d} c \int_{z}^{c} \frac{c^{\prime} \mathrm{d} c^{\prime}}{\sqrt{\left(c^{2}-c^{2}\right)\left(c^{\prime 2}-z^{2}\right)}}
$$

let $t=\left(c^{2}-c^{\prime 2}\right) /\left(c^{2}-z^{2}\right)$ and can obtain

$$
\int \frac{c^{\prime} \mathrm{d} c^{\prime}}{\sqrt{\left(c^{2}-c^{\prime 2}\right)\left(c^{\prime 2}-z^{2}\right)}}=\frac{1}{2} \int_{0}^{1} \frac{\mathrm{d} t}{\sqrt{t(1-t)}} \cdot \frac{\pi}{2}
$$

thus

$$
N(z)=\int_{z}^{\infty} n(c) \mathrm{d} c=\frac{2}{\pi} \int_{z}^{\infty} \frac{m\left(c^{\prime}\right)}{\sqrt{c^{\prime 2}-z^{2}}} \mathrm{~d} c^{\prime}
$$

where $N(z)$ is the cumulative number density of penny-shaped cracks with diameters not less than $z$ per unit volume. In order to calculate the following differentiation,

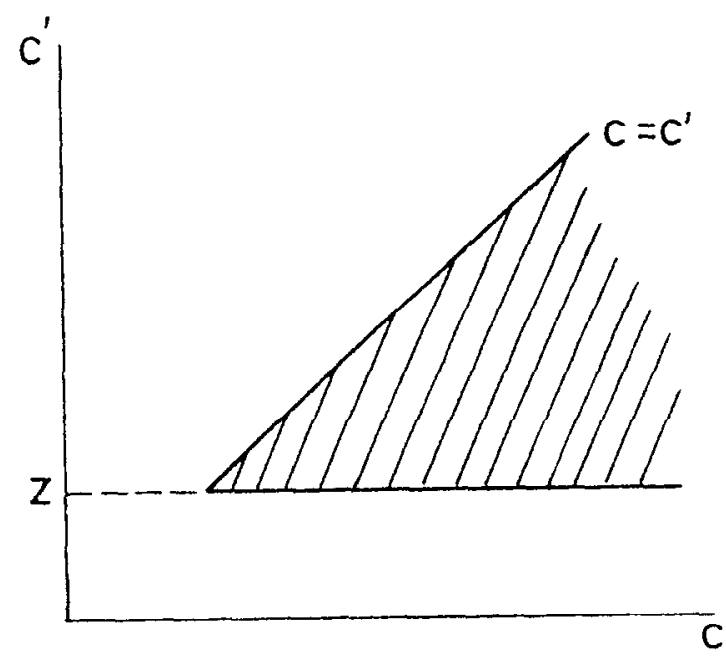

Fig. B1. The integral region of double integral (the shaded angular domain). 


$$
n(z)=-\frac{\mathrm{d} N(z)}{\mathrm{d} z}=-\frac{2}{\pi} \frac{\mathrm{d}}{\mathrm{d} z} \int_{z}^{\infty} \frac{m\left(c^{\prime}\right)}{\sqrt{c^{\prime 2}-z^{2}}} \mathrm{~d} c^{\prime}
$$

we notice

$$
\frac{\mathrm{d} c^{\prime}}{\sqrt{c^{2}-z^{2}}}=\frac{1}{c^{\prime}} \mathrm{d}\left(\sqrt{c^{\prime 2}-z^{2}}\right)
$$

and then integrate by parts, thus

$$
n(z)=\frac{2}{\pi} \int_{z}^{\infty} \frac{z}{\sqrt{c^{\prime 2}-z^{2}}}\left[-\frac{1}{c^{\prime}} \frac{\mathrm{d} m\left(c^{\prime}\right)}{\mathrm{d} c^{\prime}}+\frac{m\left(c^{\prime}\right)}{c^{\prime 2}}\right] \mathrm{d} c^{\prime}
$$

In the derivation, we have used the apparent boundary condition

$$
\lim _{i \rightarrow \infty} m\left(c^{\prime}\right)=0 .
$$

